\title{
Erratum to: Surface functionalization of nanofibrillated cellulose using click-chemistry approach in aqueous media
}

\author{
Nikolaos Pahimanolis • Ulla Hippi - Leena-Sisko Johansson • Tapio Saarinen • \\ Nikolay Houbenov $\cdot$ Janne Ruokolainen · Jukka Seppälä
}

Published online: 31 October 2017

(C) The Author(s) 2017. This article is an open access publication

\section{Erratum to: Cellulose (2011) 18:1201-1212 DOI 10.1007/s10570-011-9573-4}

The article "Surface functionalization of nanofibrillated cellulose using click-chemistry approach in aqueous media", written by Nikolaos Pahimanolis, Ulla Hippi, Leena-Sisko Johansson, Tapio Saarinen, Nikolay Houbenov, Janne Ruokolainen and Jukka Seppälä, was originally published Online First without open access. After publication in volume 18, issue 5, pages 1201-1212, the author decided to opt for Open
Choice and to make the article an open access publication. Therefore, the copyright of the article has been changed to (C) The Author(s) 2017 and the article is forthwith distributed under the terms of the Creative Commons Attribution 4.0 International License (http://creativecommons.org/licenses/by/4.0/), which permits use, duplication, adaptation, distribution and reproduction in any medium or format, as long as you give appropriate credit to the original author(s) and the source, provide a link to the Creative Commons license, and indicate if changes were made.

The online version of the original article can be found under doi:10.1007/s10570-011-9573-4.

N. Pahimanolis · U. Hippi · T. Saarinen · J. Seppälä ( $ه)$ Department of Biotechnology and Chemical Technology, Aalto University School of Chemical Technology,

P.O. Box 16100, Aalto, Espoo, Finland

e-mail: jukka.seppala@tkk.fi

L.-S. Johansson

Department of Forest Products Technology, Aalto

University School of Chemical Technology,

P.O. Box 16400, Aalto, Espoo, Finland

N. Houbenov · J. Ruokolainen

Department of Applied Physics, Aalto University School

of Science, P.O. Box 15100, Aalto, Espoo, Finland 